\title{
UNIFIED TREATMENT OF ONE- AND TWO-CENTER ONE-RANGE ADDITION THEOREMS FOR COMPLETE ORTHONORMAL SETS OF SELF-FRICTIONAL EXPONENTIAL TYPE ORBITALS AND NONINTEGER $n$ SLATER FUNCTIONS
}

\author{
I.I. GUSEINOV \\ Department of Physics, Faculty of Arts and Sciences, Onsekiz Mart University, Çanakkale, Turkey
}

\begin{abstract}
Using $L^{\left(\alpha^{*}\right)}$-self-frictional Laguerre polynomials $\left(L^{\left(\alpha^{*}\right)}\right.$-SFLPs) introduced by the author in standard convention, the combined theory of one- and twocenter one-range addition theorems for complete orthonormal sets of $\psi^{\left(\alpha^{*}\right)}$-self-frictional exponential type orbitals $\left(\psi^{\left(\alpha^{*}\right)}\right.$-SFETOs) and $\chi$-noninteger $n$ Slater type orbitals ( -NISTOs) in terms of $\chi$-integer $n$ STOs ( $\chi$-ISTOs) is established, where $\alpha^{*}$ are the integer $\left(\alpha^{*}=\alpha,-\infty<\alpha \leq 2\right)$ or noninteger ( $\alpha^{*} \neq \alpha,-\infty<\alpha<3$ ) SF quantum numbers. The expansion coefficients of these one-range addition theorems are expressed through the overlap integrals of $\chi$ -NISTOs.
\end{abstract}

Keywords: Addition theorems, Self-frictional field, Laguerre polynomials, Exponential type orbitals

\section{INTRODUCTION}

It is well known that the Laplace expansion of the Coulomb potential

$$
\frac{1}{r_{21}}=\sum_{l=0}^{\infty} \sum_{m=-l}^{l} \frac{4 \pi}{2 l+1} \frac{r_{<}^{l}}{r_{>}^{l+1}} S_{l m}^{*}\left(\theta_{2}, \phi_{2}\right) S_{l m}\left(\theta_{1}, \phi_{1}\right)
$$

possesses a characteristic two-range form, where $r_{<}=\min \left(r_{1}, r_{2}\right)$ , $r_{>}=\max \left(r_{1}, r_{2}\right)$ [1]. Here, $S_{l m}$ is the complex (for $S_{l m} \equiv Y_{l m}$ ) or real spherical harmonic. We note that the definition of phases for the complex spherical harmonics used in this work $\left(Y^{*}=Y_{l-m}\right)$ (see Ref. [2]) differs from the Condon-Shortley phases [3] by the sign factor $(-1)^{m}$. The during application, the Laplace-like two-range addition theorems lead to nontrivial technical problems. It is highly desirable to use the one-range addition theorems as they are capable to simplify subsequent integrations in multicenter integrals substantially. It should be noted that the one-range addition theorems are essentially expansions of a given function belonging to a suitable Hilbert space in terms of functions that are complete and orthonormal in this Hilbert space. Accordingly, it is desirable to use the one-range addition theorems for $\psi^{\left(\alpha^{*}\right)}$ -SFETOs that are a large class of complete and orthonormal basis functions in corresponding Hilbert spaces. In this case, the problems associated with the evaluation of multicenter integrals do not arise (see Refs. [4,5]).

The aim of this paper is, using relation for $L^{\left(\alpha^{*}\right)}$-SFLPs suggested by the author in standard convention (see Ref. [6]) and references to our works on standard convention), the combined formulas for one- and two-center onerange addition theorems for the complete orthonormal sets of $\psi^{\left(\alpha^{*}\right)}$-SFETOs and for $\chi$-NISTOs and $\chi$-ISTOs.

2. Basic formulas

The ETOs used in this work are defined as follows [5]:

for $\psi^{\left(\alpha^{*}\right)}$-SFETOs

$\psi_{n l m}^{\left(\alpha^{*}\right)}(\zeta, \vec{r})=R_{n l}^{\left(\alpha^{*}\right)}(\zeta, r) S_{l m}(\theta, \phi)=(2 \zeta)^{\frac{3}{2}} R_{n l}^{\left(\alpha^{*}\right)}(x) S_{l m}(\theta, \phi)$

$R_{n l}^{\left(\alpha^{*}\right)}(x)=e^{-\frac{x}{2}} L_{n l}^{\left(\alpha^{*}\right)}(x)$

$L_{n l}^{\left(\alpha^{*}\right)}(x)=\frac{1}{\Gamma\left(p_{l}^{*}+1\right)}\left[\frac{\Gamma\left(q_{n}^{*}+1\right)}{(2 n)^{\alpha^{*}}(n-(l+1)) !}\right] x_{1}^{l} F_{1}\left(-[n-(l+1)] ; p_{l}^{*}+1 ; x\right)=\sum_{n=l+1}^{n} \tilde{\omega}_{n \eta}^{\left(\alpha^{*}\right) !} x^{n-1}$

$\tilde{\omega}_{n \eta}^{\left(\alpha^{*}\right) l}=\left[\frac{(2 \eta) ! \Gamma\left(q_{n}^{*}+1\right)}{(2 n)^{\alpha^{*}}(n-(l+1)) !}\right]^{\frac{1}{2}} \tilde{a}_{n \eta}^{\left(\alpha^{*}\right) l}$

$$
\tilde{a}_{n \eta}^{\left(\alpha^{*}\right) l}=\frac{(-[n-(l+1)])_{\eta-(l+1)}}{\Gamma\left(p_{l}^{*}+1\right)\left(p_{l}^{*}+1\right)_{\eta-(l+1)}(\eta-(l+1)) !},
$$

for $\chi$-NISTOs

$$
\chi_{n^{*} / m}(\zeta, \vec{r})=R_{n^{*}}(\zeta, r) S_{l m}(\theta, \phi)
$$

$$
R_{n^{*}}(\zeta, r)=\frac{(2 \zeta)^{n^{*}+\frac{1}{2}}}{\left[\Gamma\left(2 n^{*}+1\right)\right]^{\frac{1}{2}}} r^{n^{*}-1} e^{-\zeta r}
$$

where $x=2 \zeta r, \quad p_{l}{ }^{*}=2 l+2-\alpha^{*}, q_{n}{ }^{*}=n+l+1-\alpha^{*}$ and ${ }_{1} F_{1}$ is the confluent hypergeometric function; $(-[n-(l+1)])_{\eta-(l+1)}$ and $\left(p_{l}{ }^{*+1)_{\eta-(l+1)}}\right.$ are the Pochhammer symbols [7].

The orthogonality relations are defined as [5]

$$
\begin{aligned}
& \text { for } \psi^{\left(\alpha^{*}\right)} \text {-SFETOS } \\
& \int \psi_{n l m}^{\left(\alpha^{*}\right)^{*}}(\zeta, \vec{r})\left(\frac{2 n}{2 \zeta r}\right)^{\alpha^{*}} \psi_{n^{\prime} l^{\prime}}^{\left(\alpha^{*}\right)}(\zeta, \vec{r}) d^{3} \vec{r}=\delta_{m m^{\prime}} \delta_{l l^{\prime}} \delta_{m n^{\prime}}, \\
& \text { for } \chi \text {-NISTOs } \\
& \int \chi_{n^{*} l m}^{*}(\zeta, \vec{r}) \chi_{n^{*} l^{\prime} m^{\prime}}(\zeta, \vec{r}) d^{3} \vec{r}=\frac{\Gamma\left(n^{*}+n^{\prime *}+1\right)}{\left[\Gamma\left(2 n^{*}+1\right) \Gamma\left(n^{\prime *}+1\right)\right]^{\frac{1}{2}}} \delta_{l l^{\prime}} \delta_{m m^{\prime}} .
\end{aligned}
$$

3. Combined one- and two-center one-range addition theorems for $\psi^{\left(\alpha^{*}\right)}$-SFETOs and $\chi$-NISTOs

Using orthogonality relations (9) and (10) and formula [5]

$$
\psi_{\mu \nu \sigma}^{\left(\alpha^{*}\right)}(\zeta, \vec{r})=\sum_{n=v+1}^{\mu} \tilde{\omega}_{\mu n}^{\left(\alpha^{*}\right) v} \chi_{n v \sigma}(\zeta, \vec{r})
$$

it is easy to obtain for the one-range addition theorems the following series expansion relations:

for $\psi^{\left(\alpha^{*}\right)}-$ SFETOS

$$
\psi_{u v s}^{\left(\alpha^{*}\right)}\left(\beta, \vec{r}_{b}\right)
$$




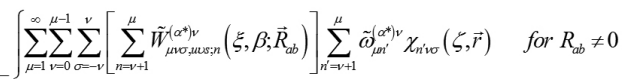

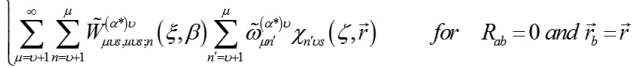

$$
\begin{aligned}
& \tilde{W}_{\mu v \sigma, u v u s ; n}^{\left(\alpha^{*}\right)}\left(\xi, \beta ; \vec{R}_{a b}\right)
\end{aligned}
$$

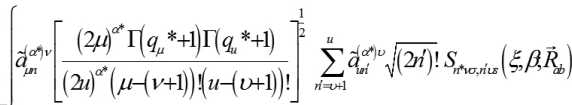

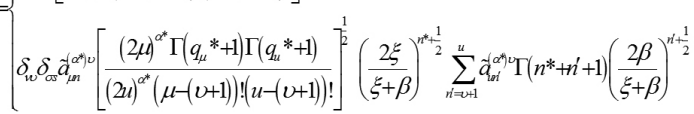

for $\chi$-NISTOs

$$
\chi_{u^{*}+s}\left(\beta, \vec{r}_{b}\right)
$$

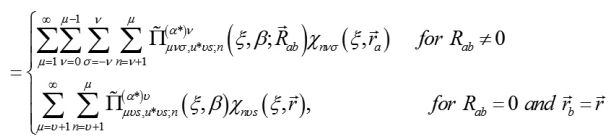

$$
\begin{aligned}
& \tilde{\Pi}_{\mu v \sigma, u^{*} v s ; n}^{\left(\alpha^{*}\right)}\left(\xi, \beta ; \vec{R}_{a b}\right)
\end{aligned}
$$

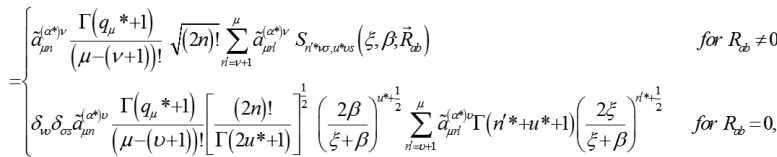

where, $n^{*}=n-\alpha^{*}$, $q_{u}^{*}=u+v+1-\alpha^{*}$ and

$$
S_{n^{*} v \sigma, u^{*} v s}\left(\xi, \beta ; \vec{R}_{a b}\right)=\sqrt{\Gamma\left(2 n^{*}+1\right)} \int \chi_{n^{*} v \sigma}^{*}\left(\xi, \vec{r}_{a}\right) \chi_{u^{*} v s}\left(\beta, \vec{r}_{b}\right) d^{3} \vec{r} .
$$

The calculation procedure of overlap integrals with $\chi$-NISTOs occurring in (20) is described in a previous paper [8].

Thus, we have established a large number of combined one- and twocenter one-range addition theorems for $\psi^{\left(\alpha^{*}\right)}$-SFETOs and $\chi$-NISTOs through the overlap integrals of $\chi$-NISTOs.

\section{Application}

As an application, we investigate the variations of scaling parameter $\zeta$ and $\mathrm{SF}$ quantum number $\alpha^{*}$ using $\psi^{\left(\alpha^{*}\right)}$-SFETOs as basis function in the Combined Hartree-Fock-Roothaan (CHFR) [9] calculations within the minimal basis set framework. The calculations have been carried out for the ground states of the some closed and open shell atoms. The CHFR total energy values obtained with minimal basis set of $\psi^{\left(\alpha^{*}\right)}$-SFETOs are given in Table 1. As can be seen from this table, the $\psi^{\left(\alpha^{*}\right)}$-SFETOs give sufficiently better energies than those obtained by the use of $\chi$-ISTOs presented in Ref. [10].

\section{CONCLUSION}

In this work, the combined one- and two-center one-range addition theorems for the complete orthonormal sets of $\psi^{\left(\alpha^{*}\right)}$-SFETOs and $\chi$ -NISTOs are presented. The origin of these theorems is based on the extension of damping or self-frictional field theory introduced by Lorentz in classical electrodynamics [11-13] to the quantum case. The suggested in this study approach is of interest for the boarder multidisciplinary areas, which span over fields as diverse as physics, chemistry, biology and mathematics.

The application to the molecular calculations based on the use of presented one-range addition theorems will be examined in future studies, when $\psi^{\left(\alpha^{*}\right)}$ -SFETOs and $\chi$-NISTOs are employed as basis functions in the CHFR theory.

\section{REFERENCES}

1. I. P. Grant, Relativistic Quantum Theory of Atoms and Molecules, Springer and Business Media, New York (2007).

2. I. I. Guseinov, J. Phys. B, 3 (1970) 1399.

3. E. U. Condon and G. H. Shortley, Theory of Atomic Spectra (Cambridge: Cambridge University Press).

4. I. I. Guseinov, In: S.A. Cetin, I. Hikmet (eds.), Sixth International Conference of the Balkan Physical Union, American Institute of Physics Conference Proceeding, 899 (2007) 65.

5. I. I. Guseinov, Bull. Chem. Soc Jpn., 85 (2012) 1306.

6. B. A. Mamedov, Int. J. Quantum Chem., 114 (2014) 361.

7. W. Magnus, F. Oberhettinger, R.P. Soni, Formulas and Theorems for the Special Functions of Mathematical Physics, New York, Springer (1966).

8. I. I. Guseinov, B.A. Mamedov, Bull. Chem. Soc Jpn., 87 (2014) 614.

9. I. I. Guseinov, J. Math. Chem., 42 (2007) 177.

10. T.Koga, J. Mol. Struct. (Theochem), 451 (1998) 1.

11. H. A. Lorentz, The theory of electrons, New York, (1953).

12. W. Heitler, The quantum theory of radiation, Oxford University Press (1950).

13. L. D. Landau, E. M. Lifshitz, The classical theory of fields, New York (1987).

Table 1. Comparison of the $\psi^{\left(\alpha^{*}\right)}$-SFETOs and $\chi$-ISTOs basis sets CHFR energies (sign reversed) for the ground states of atoms (in a.u.).

\begin{tabular}{|c|c|c|r|c|}
\hline & \multicolumn{3}{|c|}{$\psi^{\left(\alpha^{*}\right)}$-MGETOs } & $\chi$-ISTOs \\
\hline Atoms & $\zeta$ & $\alpha^{*}$ & $E^{\left(\alpha^{*}\right)}$ & E (Ref. [10]) \\
\hline$B e\left({ }^{1} S\right)$ & 3.691407070 & 2.569162410 & 14.55884765 & 14.55673986 \\
\hline$B\left({ }^{2} P\right)$ & 4.687950979 & 2.587367937 & 24.50118708 & 24.49836862 \\
\hline$C\left({ }^{3} P\right)$ & 5.682340399 & 2.620050992 & 37.62565070 & 37.62238861 \\
\hline$N\left({ }^{4} S\right)$ & 6.675584958 & 2.651592295 & 54.27248239 & 54.26889958 \\
\hline$O\left({ }^{3} P\right)$ & 7.669506754 & 2.665079300 & 74.54457490 & 74.54036465 \\
\hline$F\left({ }^{2} P\right)$ & 8.662668292 & 2.681211717 & 98.94683125 & 98.94211325 \\
\hline$N e\left({ }^{1} S\right)$ & 9.655318597 & 2.697527794 & 127.8173223 & 127.8121810 \\
\hline
\end{tabular}

